\title{
The Quantum Group Structure Associated with Non-Linearly Extended Virasoro Algebras
}

\section{Eugène Cremmer and Jean-Loup Gervais}

Laboratoire de Physique Théorique de l'École Normale Supérieure ${ }^{\star}, 24$, rue Lhomond, F-S 75231 Paris Cedex 05, France

Received November 12, 1989

\begin{abstract}
Recently, an infinite family of chiral Virasoro vertex operators, whose exchange algebra is given by the universal $R$-matrix of $S L(2)_{q}$, has been constructed. In the present paper, the case of non-linearly $(W$-) extended Virasoro symmetries, related to the algebras $A_{N}, N>1$, is considered along the same line. Contrary to the previous case $\left(A_{1}\right)$ the standard $R$-matrix of $S L(N+1)_{q}$ does not come out, and a different solution of Yang and Baxter's equations is derived. The associated quantum group structure is displayed.
\end{abstract}

Quantum groups seem to underlie the very basic structure of conformal field theories. In particular, for the Virasoro family (2D gravity and minimal models) a precise connection has just been established $[1,2]$. The method is to relate the monodromy properties of the differential equation satisfied by the chiral vertex operators to the $R$-matrix of $S L(2)_{q}$. The first step in this direction was taken by Babelon [1] who established such a relationship for the simplest $J=1 / 2$ representation. This was fully generalized by one of us (J.-L. G.) [2] who recently constructed an infinite family of chiral vertex operators whose exchange algebra is given by the universal $R$-matrix of $S L(2)_{q}$. This Virasoro family is intrinsically related to the simple algebra $A_{1}$, since it may be generated from Liouville theory which is the $A_{1}$ Toda field theory [3], and includes the $\left(S U(2)_{k} \otimes S U(2)_{1}\right) / S U(2)_{k+1}$ coset models. There are more general conformal families of the same type [3-5] with non-linearly ( $W$-) extended Virasoro algebras, which are associated with the higher simple Lie algebras $[3,5]$. In the present paper, we begin the extension of the construction carried out in $[1,2]$ to these models, by deriving an exchange algebra for the simplest set of chiral operators (in the defining representation) similar to the spin 1/2 case worked out in [1]. It will be shown that contrary to the Virasoro family, the standard $R$-matrix of the associated quantum group does not

* Unité Propre du Centre National de la Recherche Scientifique, associée à l'École Normale Supérieure et à l'Université de Paris-Sud 
come out and that different solutions of Yang and Baxter's equations are derived. Thus the quantum group structure associated with $W$-algebras seems to be new.

As shown by Bilal and Gervais [5], one may associate a $W$-algebra with any simple Lie algebra. We shall restrict ourselves to $A_{N}$ for simplicity (the generalization to other simply-laced algebras seems straightforward). In [5] the following exchange algebra was derived. There exist $N+1$ fields on the unit circle which satisfy

$$
V_{j}(\sigma) V_{k}\left(\sigma^{\prime}\right)=\sum_{\substack{l=1, \ldots, N+1 \\ m=1, \ldots, N+1}} R_{j k}^{l m}(\boldsymbol{\varpi}) V_{l}\left(\sigma^{\prime}\right) V_{m}(\sigma) .
$$

$\sigma$ is the coordinate variable on the circle $z=e^{i \sigma}$. For completeness some details of [5] are given in the appendix. The only non-vanishing $R_{j k}^{l m}$ s are:

$$
\begin{gathered}
R_{j j}^{j j}=e^{-i h \in N /(N+1)}, \quad R_{j k}^{j k}=\frac{\sin h}{\sin h \varpi_{j k}} e^{i h \in\left(1 /(N+1)-\varpi_{k l}\right)}, \\
R_{j k}^{k j}=\frac{\Gamma\left(\varpi_{j k} h / \pi\right) \Gamma\left(1+\varpi_{j k} h / \pi\right)}{\Gamma\left(1+\left(\varpi_{j k}+1\right) h / \pi\right) \Gamma\left(\left(\varpi_{j k}-1\right) h / \pi\right)} e^{i h \varepsilon /(N+1)} .
\end{gathered}
$$

$\varepsilon$ is equal to the $\operatorname{sign}^{1}$ of $\sigma-\sigma^{\prime} . h$ is a parameter whose relation with the Virasoro central charge is displayed on Eq. (A.17, 18). Introducing vectors in the space of $A_{N}$ weights, one may write $\varpi_{j k}$ under the form

$$
\varpi_{j k}=\left(\lambda^{(k)}-\lambda^{(j)}\right) \cdot \boldsymbol{\varpi},
$$

where $\lambda^{(j)}$ are the weights of the defining representation. The vector $\boldsymbol{\omega}$ is introduced in the appendix. It is a linear combination of zero-modes of free fields. These are shifted by the $V_{k}$ fields so that, for any function $f$, one has ${ }^{2}$

$$
V_{j} f(\boldsymbol{\sigma})=f\left(\boldsymbol{\varpi}+\lambda^{(j)}\right) V_{j}
$$

Next, it is convenient to replace the $V$-fields by other fields denoted by $\psi$ which are of the form $\psi_{k} \equiv d_{k}(\boldsymbol{\varpi}) V_{k}$. The $d_{k}(\boldsymbol{\varpi})$ 's are such that the exchange matrix of the $\psi_{k}$ 's which we denote by $S_{j k}^{l m}$ only involves trigonometric functions. One has:

Obviously

$$
S_{j k}^{l m}=\frac{d_{j}(\boldsymbol{\varpi}) d_{k}\left(\boldsymbol{\omega}+\lambda^{(j)}\right)}{d_{l}(\boldsymbol{\varpi}) d_{m}\left(\boldsymbol{\sigma}+\lambda^{(l)}\right)} R_{j k}^{l m} .
$$

$$
R_{j k}^{j k}=S_{j k}^{j k} .
$$

Due to the special form of $R$ the only change of the exchange matrix is for the matrix elements

$$
S_{j k}^{k j}=\frac{d_{j}(\boldsymbol{\varpi}) d_{k}\left(\boldsymbol{\varpi}+\lambda^{(j)}\right)}{d_{k}(\boldsymbol{\varpi}) d_{j}\left(\boldsymbol{\varpi}+\lambda^{(k)}\right)} R_{j k}^{k j} .
$$

There is a one-parameter family of solutions

$$
d_{j}(\boldsymbol{\varpi})=\prod_{r \neq j}\left(\Gamma\left(-\boldsymbol{\varpi}_{j r} h / \pi\right)\right)^{a} \prod_{s \neq j}\left(\Gamma\left(\left(\boldsymbol{\varpi}_{j s}-1\right) h / \pi\right)\right)^{1-a},
$$

1 To simplify we only deal with the half circle $0 \leqq \sigma \leqq \pi$ explicitly, so that $\left|\sigma-\sigma^{\prime}\right| \leqq \pi$, and $\varepsilon$ is well defined

2 No summation over repeated indices is understood unless explicitly indicated 
where $a$ is arbitrary. The new exchange matrix reads

$$
\begin{gathered}
S_{j j}^{j j}=e^{-i h \in N /(N+1)}, \quad S_{j k}^{j k}(\boldsymbol{\varpi})=\frac{\sin h}{\sin \left(h \varpi_{j k}\right)} e^{i h \in\left(1 /(N+1)-\varpi_{j k}\right)}, \\
S_{j k}^{k j}(\boldsymbol{\varpi})=\frac{\sin \left(h\left(\varpi_{j k}+1\right)\right)}{\sin \left(h \varpi_{j k}\right)} e^{i h \in /(N+1)} .
\end{gathered}
$$

Since they describe the exchange properties of the $\psi$, the $S_{j k}^{l m}$ 's automatically satisfy Yang and Baxter's equations. According to (4), however these relations take the form:

$$
\begin{aligned}
& \sum_{\varrho, \tau, \mu} S_{i \tau}^{l \varrho}\left(\boldsymbol{\varpi}, \sigma_{1}-\sigma_{3}\right) S_{j k}^{\tau \mu}\left(\boldsymbol{\varpi}+\lambda^{(i)}, \sigma_{2}-\sigma_{3}\right) S_{\varrho \mu}^{m n}\left(\boldsymbol{\varpi}+\lambda^{(l)}, \sigma_{1}-\sigma_{2}\right) \\
& =\sum_{\varrho, \tau, \mu} S_{i j}^{\mu \tau}\left(\boldsymbol{\varpi}, \sigma_{1}-\sigma_{2}\right) S_{\tau k}^{\rho m}\left(\boldsymbol{\varpi}+\lambda^{(\mu)}, \sigma_{1}-\sigma_{3}\right) S_{\mu \varrho}^{l n}\left(\boldsymbol{\varpi}, \sigma_{2}-\sigma_{3}\right) .
\end{aligned}
$$

This expression is unusual since the $S$ matrix elements do not commute with the $\psi$-fields. Following $[1,2]$, we next look for a change of basis among the $\psi$-fields such that this $\boldsymbol{\varpi}$-dependence of the exchange matrix disappears. In this way we shall be back to the standard framework of quantum group without the ๘-variable. Introduce

$$
\xi_{\alpha}=\sum_{i=1, \ldots, N+1} u_{\alpha}^{i}(\boldsymbol{\sigma}) \psi_{i} \text { for } \alpha=1,2, \ldots, N+1
$$

The $u_{\alpha}^{i}(\boldsymbol{\varpi})$ will be determined so that the $\xi$-fields satisfy an exchange algebra

$$
\xi_{\alpha}(\sigma) \xi_{\beta}\left(\sigma^{\prime}\right)=\sum_{\gamma, \delta} \varrho_{\alpha \beta}^{\gamma \delta} \xi_{\gamma}\left(\sigma^{\prime}\right) \xi_{\delta}(\sigma),
$$

where $\varrho_{\alpha \beta}^{\gamma \delta}$ does not depend upon $\boldsymbol{\sigma}$. According to Eq. (4) we have to solve the equation

$$
\sum_{\gamma, \delta} \varrho_{\alpha \beta}^{\gamma \delta} u_{\gamma}^{j}(\boldsymbol{\varpi}) u_{\delta}^{k}\left(\boldsymbol{\varpi}+\lambda^{(j)}\right)=\sum_{l, m} S_{l m}^{j k}(\boldsymbol{\varpi}) u_{\alpha}^{l}(\boldsymbol{\varpi}) u_{\beta}^{m}\left(\boldsymbol{\varpi}+\lambda^{(l)}\right) .
$$

This shows that $\varrho$ and $S$ considered as $(N+1)^{2} \times(N+1)^{2}$ matrices have the same eigenvalues and therefore that the eigenvalues of $S$ must be independent of $\boldsymbol{m}$. This is easily checked. Indeed, one finds that $S$ has only two different eigenvalues $e^{-i h \in N /(N+1)}$ and $-e^{i h \in(N+2) /(N+1)}$ which coincide with the eigenvalues of the standard $R$-matrix of SL $(\mathrm{N}+1)_{q}$ (see below). Thus the matrix $S$ is related to the latter by a change of basis. The corresponding matrix cannot be put under the factorized form $u_{\gamma}^{j}(\boldsymbol{\sigma}) u_{\delta}^{k}\left(\boldsymbol{\sigma}+\lambda^{(j)}\right)$ which appears in (13), however, as the rest of the discussion will show. Let

$$
u_{\alpha}^{j}(\boldsymbol{\varpi}) u_{\beta}^{k}\left(\boldsymbol{\varpi}+\lambda^{(j)}\right) \equiv u_{\alpha \beta}^{j k}(\boldsymbol{\varpi})
$$

so that (13) reads, for $j \neq k$,

$$
\sum_{\gamma, \delta} \varrho_{\alpha \beta}^{\gamma \delta} u_{\gamma \delta}^{j k}(\boldsymbol{\varpi})=S_{j k}^{j k}(\boldsymbol{\varpi}) u_{\alpha \beta}^{j k}(\boldsymbol{\varpi})+S_{k j}^{j k}(\boldsymbol{\varpi}) u_{\alpha \beta}^{k j}(\boldsymbol{\varpi})
$$

Since $S_{j k}^{j k}$ and $S_{k j}^{j k}$ have poles at $\boldsymbol{\sigma}=0$, the $u_{\alpha \beta}^{j k}(\boldsymbol{\sigma})$ 's must be the matrix elements of a $(N+1)^{2} \times(N+1)^{2}$ matrix which is singular at this point. We shall nevertheless look for a solution for which these matrix elements are regular at $\boldsymbol{\sigma}=0$. Since $\varrho_{\alpha \beta}^{\gamma \delta}$ is 
independent of $\boldsymbol{\varpi}$, the left member of $(15)$ is regular at $\boldsymbol{\sigma}=0$. On the right-hand side, the residues of the poles of $S_{j k}^{j k}$ and $S_{k j}^{j k}$ must cancel and one obtains the relation:

$$
u_{\alpha \beta}^{j k}(0)=u_{\alpha \beta}^{k j}(0) \text {. }
$$

It is easy to check that this implies the vanishing of $\operatorname{det}\left(u_{\alpha \beta}^{j k}(\boldsymbol{\sigma})\right)$ at $\boldsymbol{\sigma}=0$, as anticipated. Extrapolating the results obtained for $S L(2)_{q}$, we shall make the following ansatz for $u_{\alpha}^{i}$ :

$$
u_{\alpha}^{j}=\exp \left(\mathbf{A}_{\alpha}^{j} \cdot \boldsymbol{\varpi}+B_{\alpha}^{j}\right)
$$

It follows from (16) that

$$
\mathbf{A}_{\alpha}^{j} \cdot \lambda^{(k)}=\mathbf{A}_{\alpha}^{k} \cdot \lambda^{(j)} ; \quad \text { and } \quad B_{\alpha}^{j}+B_{\beta}^{k}=B_{\alpha}^{k}+B_{\beta}^{j},
$$

which implies that $B_{\alpha}^{j}=B_{\alpha}+C^{j}$. An important consequence of the ansatz combined with (16) is that

$$
u_{\alpha \alpha}^{j k}(\boldsymbol{\varpi})=u_{\alpha \alpha}^{k j}(\boldsymbol{\varpi}) \forall \boldsymbol{\varpi}
$$

Equivalently, we shall write:

$$
u_{\alpha}^{j} \equiv \exp \left(X_{\alpha}^{j}\right) ; \quad X_{\alpha}^{j}(\boldsymbol{\sigma})+X_{\alpha}^{k}\left(\boldsymbol{\sigma}+\lambda^{(j)}\right)=X_{\alpha}^{k}(\boldsymbol{\sigma})+X_{\alpha}^{j}\left(\boldsymbol{\sigma}+\lambda^{(k)}\right) .
$$

This zero-curvature condition has the general solution:

$$
X_{\alpha}^{j}(\boldsymbol{\varpi})=Y_{\alpha}\left(\boldsymbol{\varpi}+\lambda^{(j)}\right)-Y_{\alpha}(\boldsymbol{\varpi})+C_{\alpha}^{j},
$$

where $C_{\alpha}^{j}$ are arbitrary constants. Going back to (11), and making use of Eq. (4), we arrive at an equivalent form of the transformation which we shall use from now on:

$$
\xi_{\alpha}(\sigma)=e^{-Y_{\alpha}(\sigma)}\left(\sum_{j} \psi_{j}(\sigma) e^{C_{\alpha}^{j}}\right) e^{Y_{\alpha}(\varpi)}
$$

Consider first the exchange algebra with $\alpha=\beta$. One obtains:

$$
\begin{aligned}
\xi_{\alpha}(\sigma) \xi_{\alpha}\left(\sigma^{\prime}\right)= & e^{-Y_{\alpha}}\left(\sum_{j} e^{2 C_{\alpha}^{j}} S_{j j}^{j j} \psi_{j}\left(\sigma^{\prime}\right) \psi_{j}(\sigma)\right) e^{Y_{\alpha}} \\
& +e^{-Y_{\alpha}}\left(\sum_{j \neq k} e^{C_{\alpha}^{j}+C_{\alpha}^{k}}\left(S_{j k}^{j k}+S_{k j}^{j k}\right) \psi_{j}\left(\sigma^{\prime}\right) \psi_{k}(\sigma)\right) e^{Y_{\alpha}} .
\end{aligned}
$$

It follows from the explicit expression Eq. (9) that

$$
S_{j k}^{k j}(\boldsymbol{\sigma})+S_{k j}^{k j}(\boldsymbol{\sigma})=S_{j j}^{j j}=e^{-i h \in N /(N+1)} .
$$

Thus we find

$$
\xi_{\alpha}(\sigma) \xi_{\alpha}\left(\sigma^{\prime}\right)=\varrho_{\alpha \alpha}^{\alpha \alpha} \xi_{\alpha}\left(\sigma^{\prime}\right) \xi_{\alpha}(\sigma) ; \quad \varrho_{\alpha \alpha}^{\alpha \alpha}=S_{j j}^{j j}
$$

Consider next the case $\alpha \neq \beta$. It is convenient to separate the terms involving $Y_{\alpha} \pm Y_{\beta}$ by writing

$$
\begin{aligned}
\xi_{\alpha}(\sigma) \xi_{\beta}\left(\sigma^{\prime}\right)= & e^{-\left(Y_{\alpha}+Y_{\beta}\right) / 2}\left[e^{-\left(Y_{\alpha}-Y_{\beta}\right) / 2}\left(\sum_{j} \psi_{j}(\sigma) e^{C_{\alpha}^{j}}\right) e^{\left(Y_{\alpha}-Y_{\beta}\right) / 2}\right] \\
& \times\left[e^{\left(Y_{\alpha}-Y_{\beta}\right) / 2}\left(\sum_{k} \psi_{k}\left(\sigma^{\prime}\right) e^{C_{\alpha}^{k}}\right) e^{-\left(Y_{\alpha}-Y_{\beta}\right) / 2}\right] e^{\left(Y_{\alpha}+Y_{\beta}\right) / 2}
\end{aligned}
$$


We are going to derive an exchange equation of the form:

$$
\begin{aligned}
& {\left[e^{-\left(Y_{\alpha}-Y_{\beta}\right) / 2}\left(\sum_{j} \psi_{j}(\sigma) e^{C_{\alpha}^{J}}\right) e^{\left(Y_{\alpha}-Y_{\beta}\right) / 2}\right]\left[e^{\left(Y_{\alpha}-Y_{\beta}\right) / 2}\left(\sum_{k} \psi_{k}\left(\sigma^{\prime}\right) e^{C \xi}\right) e^{-\left(Y_{\alpha}-Y_{\beta}\right) / 2}\right]} \\
& =\sum_{\gamma, \delta} e_{\alpha \beta}^{\gamma \delta}\left[e^{-\left(Y_{\gamma}-Y_{\delta}\right) / 2}\left(\sum_{j} \psi_{j}\left(\sigma^{\prime}\right) e^{C_{\gamma}^{j}}\right) e^{\left(Y_{\gamma}-Y_{\delta}\right) / 2}\right] \\
& \quad \times\left[e^{\left(Y_{\gamma}-Y_{\delta}\right) / 2}\left(\sum_{k} \psi_{k}(\sigma) e^{C_{\delta}^{k}}\right) e^{-\left(Y_{\gamma}-Y_{\delta}\right) / 2}\right]
\end{aligned}
$$

which only involves the differences $Y_{\gamma}-Y_{\delta}$, and thus do not completely specify $\varrho$. The sums $Y_{\gamma}+Y_{\delta}$ are determined as follows: Looking at Eq. (26) with $\alpha$ and $\beta$ replaced by $\gamma$ and $\delta$ respectively, one sees that Eq. (27) will take the form of the exchange algebra (12) for the fields $\xi$ if the terms which appear in the summation are such that

$$
Y_{\alpha}+Y_{\beta}=Y_{\gamma}+Y_{\delta},
$$

(up to an irrelevant constant $\left.c_{\alpha \beta \gamma \delta}\right)$ so that $e^{ \pm\left(Y_{\gamma}+Y_{\delta}\right) / 2}$ are common factors and may be freely multiplied on the right and on the left.

In the form (22), our solution will follow from the ansatz:

$$
Y_{\alpha}=(\boldsymbol{\sigma})^{2} A_{\alpha}+\boldsymbol{\sigma} \cdot \mathbf{D}_{\alpha} \Rightarrow \mathbf{A}_{\alpha}^{j}=2 A_{\alpha} \lambda^{(j)}, B_{\alpha}^{j}=A_{\alpha}\left(\lambda^{(j)}\right)^{2}+\lambda^{(j)} \cdot \mathbf{D}_{\alpha}+C_{\alpha}^{j},
$$

where $A_{\alpha}$ and $\mathbf{D}_{\alpha}$ are independent of $\boldsymbol{\sigma}$ and to be determined. Condition (28) leads to

$$
A_{\alpha}+A_{\beta}=A_{\gamma}+A_{\delta}, \quad \mathbf{D}_{\alpha}+\mathbf{D}_{\beta}=\mathbf{D}_{\gamma}+\mathbf{D}_{\delta} ; \quad \text { if } \quad \varrho_{\alpha \beta}^{\gamma \delta} \neq 0 .
$$

Introduce

$$
\varphi_{\alpha \beta}^{j k} \equiv C_{\alpha}^{j}+C_{\beta}^{k}+\frac{1}{2}\left(\mathbf{D}_{\alpha}-\mathbf{D}_{\beta}\right) \cdot\left(\lambda^{(j)}-\lambda^{(k)}\right)
$$

Condition (16) is equivalent to

$$
\varphi_{\alpha \beta}^{j k}=\varphi_{\alpha \beta}^{k j},
$$

whose general solution takes the form

$$
C_{j}^{\alpha}+\frac{1}{2} \mathbf{D}_{\alpha} \cdot \lambda^{(j)}=c^{j}+c_{\alpha},
$$

where $c^{j}, c_{\alpha}$ are arbitrary constants. Introduce

$$
v_{\gamma \delta} \equiv \frac{\left(A_{\gamma}-A_{\delta}\right)}{i h} .
$$

Substituting (29) into (27) shows that we are trying to verify an equation of the form

$$
\begin{aligned}
& \sum_{j}\left[\exp \left(\varphi_{\alpha \beta}^{j j}-\frac{N}{N+1} i h v_{\alpha \beta}\right) S_{j j}^{j j} \psi_{j}(\sigma) \psi_{j}\left(\sigma^{\prime}\right)\right] \\
& \quad+\sum_{j \neq k}\left[\exp \left(\varphi_{\alpha \beta}^{j k}+i h \frac{v_{\alpha \beta}}{N+1}\right)\left(S_{j k}^{j k} e^{-i h \nu_{\alpha \beta} \varpi_{j k}}+S_{k j}^{j k} e^{-i h v_{\alpha \beta} \varpi_{k j}}\right) \psi_{j}(\sigma) \psi_{k}\left(\sigma^{\prime}\right)\right] \\
& =\sum_{\gamma, \delta} \varrho_{\alpha \beta}^{\gamma \delta} \sum_{j}\left[\exp \left(\varphi_{\gamma \delta}^{j j}-\frac{N}{N+1} i h v_{\gamma \delta}\right) \psi_{j}\left(\sigma^{\prime}\right) \psi_{j}(\sigma)\right] \\
& \quad+\sum_{\gamma, \delta} \varrho_{\alpha \beta}^{\gamma \delta} \sum_{j \neq k}\left[\exp \left(\varphi_{\gamma \delta}^{j k}+i h \frac{v_{\gamma \delta}}{N+1}-i h v_{\gamma \delta} \varpi_{j k}\right) \psi_{j}\left(\sigma^{\prime}\right) \psi_{k}(\sigma)\right]
\end{aligned}
$$


Fourier analysis shows that $v_{\gamma \delta}$ is an integer. No $v_{\gamma \delta}$ may vanish, since otherwise the determinant of $u_{\alpha}^{j}$ would be zero for any $\boldsymbol{\varpi}$. Thus all $A_{\alpha}$ 's must be different. We shall consider the case $\varepsilon=1$ for definiteness ${ }^{3}$. In order to pursue we need to distinguish two cases:

1. $v_{\alpha \beta}$ Negative Integer. In this case, it follows from Eq. (9) that

$$
\begin{aligned}
& S_{j k}^{j k} e^{-i h v_{\alpha \beta} \varpi_{j k}}+S_{k j}^{j k} e^{-i h v_{\alpha \beta} \varpi_{k j}}=e^{-i h(N-1) / 2(N+1)} \\
& \quad \times\left[\sum_{r=1}^{-v_{\alpha \beta}-1} e^{i\left(2 r+v_{\alpha \beta}\right) h \pi_{j k}}\left(e^{3 i h / 2}-e^{-i h / 2}\right)+e^{i v_{\alpha \beta} h \pi_{j k}} e^{3 i h / 2}\right] .
\end{aligned}
$$

After some calculations, one verifies that Eq. (35) holds with

for

$$
\varrho_{\alpha \beta}^{\gamma \delta}=e^{-i h(N-1) / 2(N+1)} e^{2 i h v_{\alpha \gamma} /(N+1)}\left(e^{3 i h / 2}-e^{-i h / 2}\right),
$$

and

$$
v_{\gamma \delta}=-2 r-v_{\alpha \beta}, \quad \text { with } \quad r=1, \ldots,-v_{\alpha \beta}-1, v_{\alpha \beta} \leqq-2 ;
$$

$$
\varrho_{\alpha \beta}^{\beta \alpha}=e^{-i h(N-1) / 2(N+1)} e^{2 i h\left(v_{\alpha \beta}\right) /(N+1)} e^{3 i h / 2} .
$$

One finds an additional factor $e^{c_{\alpha}+c_{\beta}-c_{\gamma}-c_{\delta}}$, when one performs the calculations. It may be eliminated if one multiples $\xi_{\alpha}$ by $e^{-c_{\alpha}}$ and we leave it out. Equation (37a) has been simplified using Eq. (30) which gives $v_{\alpha \gamma}+v_{\beta \delta}=0$. Moreover, one easily sees, using again this relation that the possible values of $v_{\delta \alpha}$ and $v_{\beta \gamma}$ are:

$$
v_{\delta \alpha}=v_{\beta \gamma}=1, \ldots, v_{\beta \alpha} .
$$

All integer values of values $0 \leqq v_{\alpha \gamma}, v_{\alpha \delta} \leqq v_{\alpha \beta}$ appear.

2. $v_{\alpha \beta}$ Positive Integer. The discussion is similar:

$$
\begin{aligned}
& S_{j k}^{j k} e^{-i h v_{\alpha \beta} \sigma_{j k}}+S_{k j}^{j k} e^{-i h v_{\alpha \beta} \varpi_{k j}}=e^{-i h(N-1) / 2(N-1)} \\
& \times\left[\sum_{r=1}^{v_{\alpha \beta}-1} e^{i\left(2 r-v_{\alpha \beta}\right) h \varpi_{j k}}\left(e^{-i h / 2}-e^{3 i h / 2}\right)+e^{i v_{\alpha \beta} h \varpi_{j k}} e^{-i h / 2}\right] \\
& \varrho_{\alpha \beta}^{\nu \delta}=e^{-i h(N-1) / 2(N+1)} e^{2 i h v_{\alpha \gamma} /(N+1)}\left(e^{-i h / 2}-e^{3 i h / 2}\right)
\end{aligned}
$$

for

and

$$
v_{\gamma \delta}=-2 r+v_{\alpha \beta} ; \quad \text { with } \quad r=0, \ldots, v_{\alpha \beta}-1, v_{\alpha \beta} \geqq 2,
$$

$$
\varrho_{\alpha \beta}^{\beta \alpha}=e^{-i h(N-1) / 2(N+1)} e^{2 i h\left(v_{\alpha \beta}\right) /(N+1)} e^{-i h / 2} .
$$

The possible values of $v_{\alpha \gamma}$ and $v_{\delta \beta}$ are:

$$
v_{\alpha \gamma}=v_{\delta \beta}=0, \ldots, v_{\alpha \beta} .
$$

All integer values of values $0 \geqq v_{\alpha \gamma}, v_{\alpha \delta} \geqq v_{\alpha \beta}$ appear.

Denote $A_{\alpha} /$ ih by $v_{\alpha}$, so that $v_{\alpha \beta}=v_{\alpha}-v_{\beta}$. The differences $v_{\gamma}-v_{\delta}$ are integers, and we have seen that for any given $\alpha$ and $\beta$ all integer values of $\left|v_{\gamma \delta}\right|$ between 0 and $\left|v_{\alpha \beta}\right|$

3 The case $\varepsilon=-1$ may be treated in exactly the same way. We know that the $S$-matrix satisfies the inverse relation $S(\varepsilon=1) S(\varepsilon=-1)=1$. This is also true for the matrix $\varrho$ since $\varrho(\varepsilon=-1)$ and $S(\varepsilon=-1)$ are related by the same matrix $u_{\alpha \beta}^{j k}$ 
appear. Thus the $v_{\gamma}$ 's, which are spaced by one, span an interval $[v+1, v+N+1]$, with arbitrarily fixed $v$. By a constant transformation $V_{\alpha}^{\beta}$ of the $\xi$ 's we can relabel the indices, so that $v_{\gamma}=\gamma+v$. The parameter $v$ is irrelevant, since it may be absorbed by the transformation $\xi_{\alpha}^{\prime}=e^{-i h \dot{\omega}^{2}} \xi_{\alpha} e^{i h \omega^{2}}$ which preserves the matrix $\varrho$. Hence we drop it. This terminates the determination of the exchange matrix $\varrho$.

After a rescaling of the $\psi$-fields which leaves the $S$-matrix invariant, the $u_{\alpha}^{j}$ s take the simple form

$$
u_{\alpha}^{j}(\boldsymbol{\sigma})=e^{i h \alpha \lambda^{(j)} \cdot \boldsymbol{\omega}} \equiv\left(\mu^{(j)}\right)^{\alpha} .
$$

The determinant of such a matrix is well known: Up to a numerical factor,

$$
\operatorname{det}(u) \propto \prod_{j<k}\left(\mu^{(j)}-\mu^{(k)}\right) \prod_{l} \mu^{(l)} .
$$

Using the constraints $\sum_{j} \lambda^{(j)}=0$ which gives $\prod_{l} \mu^{(l)}=1$, one obtains

$$
\operatorname{det}(u) \propto \prod_{j<k} \sin \left(\frac{h \varpi_{j k}}{2}\right) .
$$

In a group theoretic language, we introduce ket vectors $\mid \delta>$ in the defining representation. The $\varrho$ matrix we may be written as:

$$
\varrho_{\alpha \beta}^{\gamma \delta}=e^{i h /(N+1)}(<\alpha|\otimes<\beta|) \varrho(|\delta>\otimes| \gamma>),
$$

where

$$
\begin{aligned}
\varrho \equiv & e^{-i h} \sum_{\alpha} e_{\alpha, \alpha} \otimes e_{\alpha, \alpha} \\
& +e^{-i h} \sum_{\alpha>\beta} e^{2 i h(\alpha-\beta) /(N+1)} e_{\alpha, \alpha} \otimes e_{\beta, \beta}+e^{i h} \sum_{\alpha<\beta} e^{2 i h(\alpha-\beta) /(N+1)} e_{\alpha, \alpha} \otimes e_{\beta, \beta} \\
& +\left(e^{i h}-e^{-i h}\right) \sum_{\alpha<\beta} \sum_{r=1}^{\beta-\alpha-1} e^{-2 i h r /(N+1)} e_{\alpha, \beta-r} \otimes e_{\beta, \alpha+r} \\
& +\left(e^{-i h}-e^{i h}\right) \sum_{\alpha>\beta} \sum_{r=0}^{\alpha-\beta-1} e^{2 i h r /(N+1)} e_{\alpha, \beta+r} \otimes e_{\beta, \alpha-r},
\end{aligned}
$$

where we have let in general

$$
e_{\gamma, \delta} \equiv|\gamma\rangle\langle\delta|
$$

For comparison we note that, with our conventions, the standard exchange matrix of $S L(N+1)_{q}$ reads:

$$
\bar{\varrho} \equiv e^{-i h} \sum_{\alpha} e_{\alpha, \alpha} \otimes e_{\alpha, \alpha}+\sum_{\alpha \neq \beta} e_{\alpha, \alpha} \otimes e_{\beta, \beta}+\left(e^{-i h}-e^{i h}\right) \sum_{\alpha>\beta} e_{\alpha, \beta} \otimes e_{\beta, \alpha} .
$$

The main difference between our $\varrho$-matrix and the standard one $\varrho$ is that the latter connects $\alpha \beta$ to $\alpha \beta$ and $\beta \alpha$ only, whereas the new exchange matrix connects $\alpha \beta$ to all pairs $\gamma \delta$ with $\alpha+\beta=\gamma+\delta$ (or more generally $v_{\alpha}+v_{\beta}=v_{\gamma}+v_{\delta}$ ). On the other hand, as we already noted they have the same eigenvalues and hence are related by a constant matrix:

$$
\varrho_{\alpha \beta}^{\gamma \delta}=\sum_{\alpha^{\prime} \beta^{\prime} \gamma^{\prime} \delta^{\prime}}\left(M^{-1}\right)_{\alpha \beta}^{\alpha^{\prime} \beta^{\prime}} \widetilde{\varrho}_{\alpha^{\prime} \beta^{\prime}}^{\gamma^{\prime} \delta^{\prime}} M_{\gamma^{\prime} \delta^{\prime}}^{\nu \delta}
$$

Thus we have to see whether $\widetilde{\varrho}$ would not provide an alternate solution of (13). An obvious arbitrariness of the above discussion is a constant redefinition of the $\xi^{\prime}$ : $\xi_{\alpha}^{\prime}$ 
$=\sum_{\beta} V_{\alpha}^{\beta} \xi_{\beta}$. The corresponding $\varrho$ and $\varrho^{\prime}$ matrices are related as in (49) but by a factorized matrix of the form $V_{\alpha}^{\gamma} V_{\beta}^{\delta}$. Thus we would conclude that the standard exchange matrix is a solution only if the matrix $M$ of (49) could be put under such a form. More generally, if we assume that we have two solutions $\varrho$ and $\varrho^{\prime}$ of $(13)$ with matrices $u_{\alpha}^{j}(\boldsymbol{\sigma})$ and $\left(u^{\prime}\right)_{\alpha}^{j}(\boldsymbol{\sigma})$ respectively, we can relate $\varrho$ and $\varrho^{\prime}$ by $\sum_{j k}\left(u^{\prime}\right)_{\alpha \beta}^{j k}(\boldsymbol{\sigma})$ $\left(u^{-1}\right)_{j k}^{\gamma \delta}(\boldsymbol{\sigma})$ which does not a priori correspond to a constant matrix as in (49). In order to settle the question, we directly show that the standard exchange matrix cannot be a solution of Eq. (13). From this last equation with $j=k$ we get arrive at the relation

$$
G_{\alpha \beta} \equiv \frac{u_{\alpha \beta}^{j j}(\boldsymbol{\sigma})}{u_{\beta \alpha}^{j j}(\boldsymbol{\sigma})}=\frac{\widetilde{\varrho}_{\alpha \beta}^{\beta \alpha}}{\tilde{\varrho}_{\alpha \alpha}^{\alpha \alpha}-\widetilde{\varrho}_{\alpha \beta}^{\alpha \beta}} .
$$

The factorization properties of $u_{\beta \alpha}^{j j}$, displayed on Eq. (14) imply that $G_{\alpha \beta} G_{\beta \gamma}=G_{\alpha \gamma}$ for any $\alpha \beta \gamma$. This condition is impossible to satisfy since the explicit expression (48) gives $G_{\alpha \beta}=e^{-i h}$ independent of $\alpha$ and $\beta$. The standard exchange matrix is thus ruled out. We can even make a stronger statement. Relax the precise identification with the standard exchange matrix, but still impose that $\varrho$ links $\alpha \beta$ to $\alpha \beta$ and $\beta \alpha$ only. Then we can find a solution of (13). However $\operatorname{det} u$ vanishes and it does not solve our problem, since the $\xi$-fields so defined are not linearly independent.

In the same way as the standard $R$-matrix of $S L(N+1)_{q}$ our final result takes a quasi triangular form if one multiplies it by the matrix $P_{\alpha \beta}^{\alpha^{\prime} \beta^{\prime}} \equiv \delta_{\alpha, \beta^{\prime}}, \delta_{\alpha^{\prime}, \beta}$ that interchanges the indices. For $N=4$, one obtains, for instance:

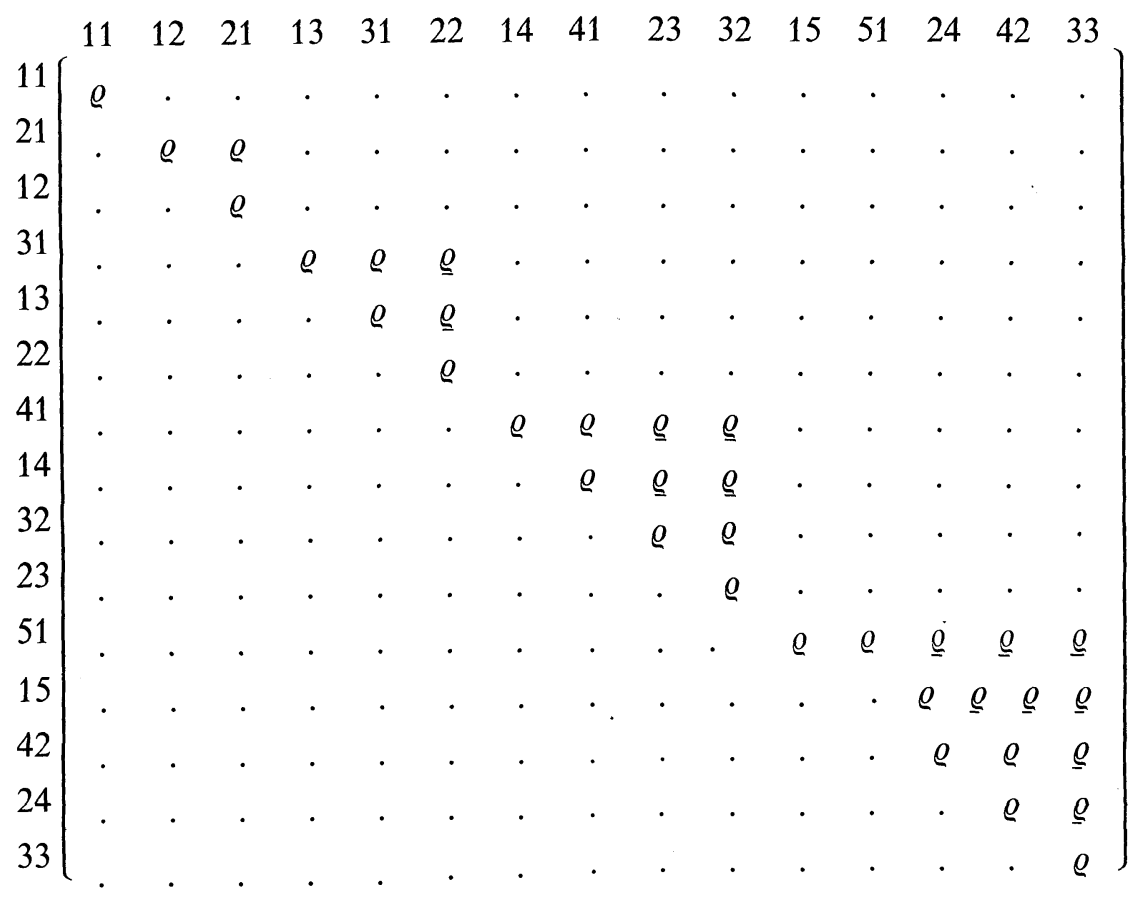


where dots indicate the vanishing matrix elements. The underlined entries are the ones which vanish for the standard $R$-matrix.

Let us finally discuss the quantum group structure associated with the exchange matrix $\varrho$. A standard method [6] is to introduce the matrix algebra:

$$
\sum_{\alpha^{\prime}, \beta^{\prime}} T_{\alpha}^{\alpha^{\prime}} T_{\beta}^{\beta^{\prime}} \varrho_{\alpha^{\prime} \beta^{\prime}}^{\gamma \delta}=\sum_{\gamma^{\prime}, \delta^{\prime}} \varrho_{\alpha \beta}^{\gamma^{\prime} \delta^{\prime}} T_{\gamma^{\prime}}^{\gamma} T_{\delta^{\prime}}^{\delta}
$$

The matrix $\varrho$ is highly degenerate since it has only two different eigenvalues. Hence there are many solutions of the equations

$$
\sum_{\alpha^{\prime}, \beta^{\prime}} \mathscr{M}_{\alpha \beta}^{\alpha^{\prime} \beta^{\prime}} \varrho_{\alpha^{\prime} \beta^{\prime}}^{\gamma \delta}=\sum_{\gamma^{\prime}, \delta^{\prime}} \varrho_{\alpha \beta}^{\gamma^{\prime} \delta^{\prime}} \mathscr{M}_{\gamma^{\prime} \delta^{\prime}}^{\gamma \delta}
$$

with $\mathscr{M}$ an ordinary $c$-number matrix. It is easy to check, nevertheless that there is no way to write $\mathscr{M}_{\alpha \beta}^{\alpha^{\prime} \beta^{\prime}}$ in the factorized form $T_{\alpha}^{\alpha^{\prime}} T_{\beta}^{\beta^{\prime}}$ if the matrix elements $T_{\alpha}^{\alpha^{\prime}}$ are assumed to be ordinary commuting numbers. Thus Eq. (52) has solutions only if the matrix elements $T_{\alpha}^{\alpha^{\prime}}$ have non-trivial commutation relations. The consistency of these commutation relations is of course ensured by the fact that $\varrho$ satisfies Yang and Baxter's equations by construction. The properties of the matrix $\varrho$ are such that Eq. (52) just describes the noncommutativity of the matrix elements and nothing more. This is a consequence of the fact that the two eigenvalues $\varrho_{0}$ and $\varrho_{1}$ have degeneracies $(N+1)(N+2) / 2$ and $N(N+1) / 2$ respectively. Therefore Eq. $(52)$ only gives

$$
(N+1)^{2}-\frac{N^{2}(N+1)^{2}}{4}-\frac{(N+1)^{2}(N+2)^{2}}{4}=\frac{(N+1)^{2}\left((N+1)^{2}-1\right)}{2}
$$

independent equations. There are just enough independent relations to reexpress the products $T_{\alpha}^{\alpha^{\prime}} T_{\alpha}^{\alpha^{\prime \prime}},\left(\alpha^{\prime}>\alpha^{\prime \prime}\right)$ and $T_{\alpha}^{\alpha^{\prime}} T_{\beta}^{\beta^{\prime}}(\alpha>\beta)$ in terms of $T_{\gamma}^{\gamma^{\prime \prime}} T_{\gamma}^{\gamma^{\prime}}, T_{\delta}^{\delta^{\prime}} T_{\gamma}^{\gamma^{\prime}}$, and $T_{\gamma}^{\gamma^{\prime}} T_{\gamma}^{\gamma^{\prime}}\left(\gamma^{\prime}>\gamma^{\prime \prime}, \gamma>\delta\right)$. This exactly defines the commutation relations of the $T_{\alpha}^{\beta}$ s. The quantum group property is clear from this construction since, given two matrices $T$ and $\widehat{T}$, with commuting matrix elements $\left(T_{\alpha}^{\beta} \widehat{T}_{\gamma}^{\delta}=\widehat{T}_{\gamma}^{\delta} T_{\alpha}^{\beta}\right)$ which satisfy Eq. (52), the product $\sum_{\beta} T_{\alpha}^{\beta} \widehat{T}_{\beta}^{\gamma}$ is also solution of the same equation. Thus the commutation relation specified by Eq. (52) define a quantum matrix algebra.

One thus sees that our exchange matrix defines a quantum deformation of $A_{N}$ that differs from the standard one if $N>1$. We have indeed worked out the simplest new cases without encountering problems. We shall not give the general result here since there are many commutation relations and the formulae become lengthy. Some details are displayed in Appendix B. For orientation let us here quote the formulae for $S L(2)$; where the present method gives the standard $R$-matrix [1], that is,

$$
\begin{gathered}
\varrho_{11}^{11}=\varrho_{22}^{22}=e^{-i h / 2}, \\
\varrho_{12}^{12}=0 ; \quad \varrho_{12}^{21}=\varrho_{21}^{12}=e^{i h / 2} ; \quad \varrho_{21}^{21}=e^{-i h / 2}-e^{3 i h / 2} .
\end{gathered}
$$

Equation (52) leads to:

$$
\begin{gathered}
a b=e^{i h} b a, c d=e^{i h} d c, c b=b c, a c=e^{i h} c a \\
b d=e^{i h} d b, a d-d a=\left(e^{i h}-e^{-i h}\right) b c, \text { for } T=\left(\begin{array}{ll}
a & b \\
c & d
\end{array}\right),
\end{gathered}
$$

in agreement with the standard expressions. 
It is also possible to define a "quantum plane" with non-commuting coordinates $x_{\alpha}$. We look for non-commutativity relations of the form

$$
x_{\alpha} x_{\beta}=\sum_{\gamma, \delta} \hat{\varrho}_{\alpha \beta}^{\gamma \delta} x_{\gamma} x_{\delta}
$$

which are preserved under the transformation

$$
x_{\alpha} \rightarrow y_{\alpha}=\sum_{\beta} T_{\alpha}^{\beta} x_{\beta},
$$

provided that

$$
x_{\gamma} T_{\alpha}^{\beta}=T_{\alpha}^{\beta} x_{\gamma} \forall \alpha, \beta, \gamma .
$$

This requirement is satisfied if we choose $\hat{\varrho}$ proportional to $\varrho$, as one easily verifies from Eq. (52). In order to ensure that (55) describes the non-commutativity of the $x$ 's without additional constraints, we must normalize $\varrho$ such that the eigenvalue $\varrho_{0}$ with multiplicity $(N+1)(N+2) / 2$ becomes equal to 1 . This then allows us to reexpress $x_{\alpha} x_{\beta}(\alpha>\beta)$ in terms of $x_{\delta} x_{\gamma}$ and $x_{\gamma} x_{\gamma}(\gamma>\delta)$. Using the expression of $\varrho_{\alpha \beta}^{\gamma \delta}$ we get after normalization

$$
\begin{aligned}
& x_{\alpha} x_{\beta}=\sum_{\gamma=\alpha+1}^{\beta-1}\left(e^{i h}-e^{-i h}\right) e^{2 i h(\alpha-\gamma) /(N+1)+i h} x_{\gamma} x_{\alpha+\beta-\gamma} \\
& +e^{2 i h(1+(\alpha-\beta) /(N+1))} x_{\beta} x_{\alpha} \text { if } \alpha<\beta ; \\
& x_{\alpha} x_{\alpha}=x_{\alpha} x_{\alpha}, \quad \text { if } \alpha=\beta \text {; } \\
& x_{\alpha} x_{\beta}=-\sum_{\gamma=\beta+1}^{\alpha}\left(e^{i h}-e^{-i h}\right) e^{2 i h(\alpha-\gamma) /(N+1)} x_{\gamma} x_{\alpha+\beta-\gamma} \\
& +e^{2 i h(\alpha-\beta) /(N+1)} x_{\beta} x_{\alpha} \quad \text { if } \alpha>\beta .
\end{aligned}
$$

On the right-hand sides of these expressions we next separate the terms $x_{\gamma} x_{\delta}$ with $\gamma<\delta$ and $\gamma>\delta$. One immediately sees that the equations for $\alpha<\beta$ and $\alpha>\beta$ are the same up to a numerical factor, and the "table of multiplication" is easily worked out. One finds:

$$
\begin{aligned}
x_{\alpha+r} x_{\alpha}= & e^{2 i h(r /(N+1)-1)} x_{\alpha} x_{\alpha+r} \\
& -\left(e^{2 i h}-e^{-2 i h)} \sum_{q=1}^{\left[\frac{r+1}{2}\right]-1} e^{2 i h((r-q) /(N+1)-q} x_{\alpha+q} x_{\alpha+r-q}\right. \\
& + \begin{cases}0, & \text { if } r \text { is odd } \\
-e^{2 i h(r /(2(N+1))+1-r / 2)}\left(1-e^{-2 i h}\right) x_{\alpha+r} x_{\alpha+r}, & \text { if } r \text { is even. }\end{cases}
\end{aligned}
$$

Let us give the simplest new example $(S L(3))$. Our result reads:

$$
\begin{aligned}
& x_{2} x_{1}=e^{-4 i h / 3} x_{1} x_{2}, \\
& x_{3} x_{1}=e^{-2 i h / 3} x_{1} x_{3}-e^{-i h / 3}\left(e^{i h}-e^{-i h}\right) x_{2} x_{2}, \\
& x_{3} x_{2}=e^{-4 i h / 3} x_{2} x_{3} .
\end{aligned}
$$

This should be compared with the quantum plane associated with the standard exchange matrix:

$$
x_{2} x_{1}=e^{-i h} x_{1} x_{2} ; \quad x_{3} x_{1}=e^{-i h} x_{1} x_{3} ; \quad x_{3} x_{2}=e^{-i h} x_{2} x_{3} .
$$




\section{Appendix A}

For completeness we recall some basic points in the derivation of the exchange algebra equation (2) carried out in [5] by solving the $A_{N}$ Toda field theory ${ }^{4}$. Consider first the classical ( $c$-number limit). The solution of the Toda field equation leads to the basic chiral differential equation:

$$
\left(-\frac{d^{N+1}}{(d \sigma)^{N+1}}+\sum_{k=2}^{N+1} T_{(k)} \frac{d^{N-k+1}}{(d \sigma)^{N-k+1}}\right) \psi_{a}=0 .
$$

The fields $T_{(k)}$ have conformal weights $k$ up to central terms. They are the generators of the $W$-algebra. This comes out as follows. There exist fields $p_{k}(\sigma)$, $k=1, \ldots, N+1$ with classical Poisson bracket structure:

$$
\left\{p_{k}\left(\sigma_{1}\right), p_{l}\left(\sigma_{2}\right)\right\}=4 \pi \mathscr{K}_{k l} \delta^{\prime}\left(\sigma_{1}-\sigma_{2}\right),
$$

$\boldsymbol{\alpha}_{k}, k=1, \ldots, N$ are a set of simple roots ${ }^{5} . \mathscr{K}_{k l}=\boldsymbol{\alpha}_{k} \cdot \boldsymbol{\alpha}_{l}$ is the Cartan matrix of $A_{N}$. A standard construction of the simple roots of $A_{N}$ is to take $N+1$ orthonormal vectors $\mathbf{e}_{r}$ and to let $\alpha_{r}=\mathbf{e}_{r}-\mathbf{e}_{r+1}, r=1, \ldots, N . T_{(2)}(\sigma)$ is the stress-energy tensor. In terms of the $p$-fields it is given by:

$$
T_{(2)}(\sigma) \equiv \gamma \sum_{k, l=1}^{N+1}\left(\mathscr{K}^{-1}\right)_{k l} p_{k}(\sigma) p_{l}(\sigma)+\sqrt{\gamma} \frac{N+1}{2} \sum_{l=1}^{N+1}\left(\mathscr{K}^{-1}\right)_{l l} p_{l}^{\prime}(\sigma)
$$

In view of (A.2) the Virasoro generators

satisfy

$$
\begin{gathered}
L_{n} \equiv \frac{1}{8 \pi \gamma} \int_{0}^{2 \pi} d \sigma T(\sigma) e^{i n \sigma}+\frac{N(N+1)(N+2)}{48 \gamma} \delta_{n, 0} \\
i\left\{L_{m}, L_{n}\right\}=(m-n) L_{m+n}+\frac{C}{12}\left(m^{3}-m\right) \delta_{m,-n},
\end{gathered}
$$

with $C=N(N+1)(N+2) / 2 \gamma \cdot \gamma$ is a free parameter that plays the role of a coupling constant. It is convenient to introduce

$$
\Lambda(\sigma) \equiv \sum_{l=1}^{N} \lambda_{l} p_{l}(\sigma)
$$

with $\lambda_{j} \cdot \boldsymbol{\alpha}_{k}=\delta_{j, k}$. The $\lambda_{j}$ are a set of fundamental weights of $A_{N}$. Equation (A.3) may be rewritten as

$$
T_{(2)}(\sigma) \equiv \gamma \boldsymbol{\Lambda} \cdot \boldsymbol{\Lambda}+\sqrt{\gamma} \sum_{l=1}^{N+1} \lambda_{l} \cdot \Lambda^{\prime}
$$

The expression of the other $T_{(k)}$ fields is derived by reexpressing (A.1) in the form:

so that

$$
\left(\frac{d}{d \sigma}-\mathbf{e}_{N+1} \cdot \boldsymbol{\Lambda}\right)\left(\frac{d}{d \sigma}-\mathbf{e}_{N} \cdot \boldsymbol{\Lambda}\right) \ldots\left(\frac{d}{d \sigma}-\mathbf{e}_{1} \cdot \boldsymbol{\Lambda}\right) \psi_{a}=0,
$$

$$
T_{(k)} \equiv(-1)^{k+1} \sum_{m_{1}>\ldots>m_{k}=1}^{N+1}\left(\mathbf{e}_{m_{1}} \cdot \boldsymbol{\Lambda}\right)\left(\mathbf{e}_{m_{2}} \cdot \boldsymbol{\Lambda}\right) \ldots\left(\mathbf{e}_{m_{k}} \cdot \mathbf{\Lambda}\right)+\ldots
$$

\footnotetext{
4 We presently use a slightly different notation which is simpler

5 Arrows denote vectors in the weight space of $A_{N}$
} 
One may verify from this expression and making use of (A.2), that the $T_{(k)}$ 's satisfy a Poisson bracket realization of a $W$-algebra.

The $p$-fields are periodic:

and it is useful to introduce $p_{k}(\sigma)=\sum_{n} p_{n}^{(k)} e^{-i n \sigma}$,

$$
\Lambda_{0} \equiv \sum_{l=1}^{N} \lambda_{l} p_{0}^{(l)}
$$

A convenient set of independent solutions of (A.1) is of the form

$$
\begin{gathered}
V_{1}(\sigma)=\exp \left(\sqrt{\gamma} \sum_{j}\left(\mathscr{K}^{-1}\right)_{1 j} \phi_{j}(\sigma)\right), \\
\phi_{j}(\sigma) \equiv q_{0}^{(j)}+p_{0}^{(j)} \sigma+i \sum_{n \neq 0} \frac{p_{n}^{(j)}}{n} e^{-i n \sigma} ; \quad \phi_{j}^{\prime}(\sigma)=p_{j}(\sigma), \\
V_{r}(\sigma)=\mathscr{S}_{r+1}(\sigma) V_{1}(\sigma) .
\end{gathered}
$$

$\mathscr{S}_{r+1}(\sigma)$ are classical screening operators such that

$$
V_{r}(\sigma+2 \pi)=\exp \left(2 \pi \Lambda_{0} \cdot \lambda^{(r)}\right) V_{r}(\sigma) .
$$

The $V_{r}$ are thus chosen to be eigenvectors of the monodromy matrix. The vectors

$$
\lambda^{(r)} \equiv \lambda_{1}-\alpha_{r}=\mathbf{e}_{r}-\frac{1}{N+1} \sum_{j=1}^{N+1} \mathbf{e}_{j}
$$

are the weights of the defining representation. $q_{0}^{(j)}$ and $p_{0}^{(k)}$ are conjugate dynamical variables:

$$
\left\{q_{0}^{(j)}, p_{0}^{(k)}\right\}=2 \mathscr{K}_{j k}
$$

The form of the solution (A.12) is not symmetric between the weights $\lambda^{(r)}$. This is only due to a particular choice of the $p$-fields. One may define equivalent $p$-fields with $V_{r} \leftrightarrow V_{s}, s=r+a \bmod N+1$. The symmetry between the $V_{k}^{\prime}$ s is actually the origin of the quantum group structure, as already shown in [2].

In the quantum case, Eq. (A.2) and (A.12a) become respectively

$$
\begin{aligned}
& {\left[p_{k}\left(\sigma_{1}\right), p_{l}\left(\sigma_{2}\right)\right]=4 \pi i \mathscr{K}_{k l} \delta^{\prime}\left(\sigma_{1}-\sigma_{2}\right),} \\
& V_{1}=:\left(\exp \left(\sqrt{\frac{h}{2 \pi}} \sum_{j}\left(\mathscr{K}^{-1}\right)_{1 j} \phi_{l}\right)\right):
\end{aligned}
$$

where there are two choices for the number $h$ :

$$
\begin{aligned}
h_{ \pm} & =\frac{\pi}{4 \gamma}(1-4 \gamma \pm \sqrt{1-8 \gamma}) \\
& =\frac{\pi}{2 N(N+1)(N+2)}\left(C-\frac{N+C_{1}}{2} \pm \sqrt{\left(C-C_{1}\right)(C-N)}\right)
\end{aligned}
$$

and $C_{1}=N(2 N+3)^{2}$. The second expression of $h$ follows from the fact that the central charge is given by

$$
C=N+\frac{N(N+1)(N+2)}{2 \gamma} .
$$


Equations (A.17) are such that an operator equation similar to (A.1) holds. The study of its monodromy properties leads to Eqs. (1) and (2) where

$$
\boldsymbol{\sigma} \equiv-\frac{i}{2} \sqrt{\frac{2 \pi}{h}} \boldsymbol{\Lambda}_{0}
$$

\section{Appendix B}

In this appendix, we shall explicitly solve the matrix algebra equation

$$
\sum_{\alpha^{\prime}, \beta^{\prime}} T_{\alpha}^{\alpha^{\prime}} T_{\beta}^{\beta^{\prime}} \hat{\varrho}_{\alpha^{\prime} \beta^{\prime}}^{\gamma \delta}=\sum_{\gamma^{\prime}, \delta^{\prime}} \hat{\varrho}_{\alpha \beta}^{\gamma^{\prime} \delta^{\prime}} T_{\gamma^{\prime}}^{\gamma} T_{\delta^{\prime}}^{\delta}
$$

Up to an overall common factor, this relation is identical to Eq. (52). Our goal is to reexpress the products $T_{\alpha}^{\alpha^{\prime}} T_{\alpha}^{\alpha^{\prime \prime}},\left(\alpha^{\prime}>\alpha^{\prime \prime}\right)$ and $T_{\alpha}^{\alpha^{\prime}} T_{\beta}^{\beta^{\prime}}(\alpha>\beta)$ in terms of the others, that is, $T_{\gamma}^{\gamma^{\prime \prime}} T_{\gamma}^{\gamma^{\prime}}, T_{\delta}^{\delta^{\prime}} T_{\gamma}^{\gamma^{\prime}}$, and $T_{\gamma}^{\gamma^{\prime}} T_{\gamma}^{\gamma^{\prime}}\left(\gamma^{\prime}>\gamma^{\prime \prime}, \gamma>\delta\right)$.

Let us first consider the case $\alpha=\beta$. Equation (B.1) reads ( $\varrho$ is such that $\left.\hat{\varrho}_{\alpha \alpha}^{\alpha \alpha}=1\right)$

$$
T_{\alpha}^{\gamma} T_{\alpha}^{\delta}=\sum_{\gamma^{\prime} \delta^{\prime}} T_{\alpha}^{\gamma^{\prime}} T_{\alpha}^{\delta^{\prime}} \hat{\varrho}_{\gamma^{\prime} \delta^{\prime}}^{\gamma \delta}
$$

Introduce $\widetilde{T}_{\alpha}^{\gamma} \equiv e^{2 i h(\gamma-\alpha) /(N+1)} T_{\alpha}^{\gamma}$. We isolate the terms with $\gamma^{\prime}>\delta^{\prime}$ and $\gamma^{\prime} \leqq \delta^{\prime}$, obtaining, for $\gamma>1$,

$$
\widetilde{T}_{\alpha}^{\delta} T_{\alpha}^{\gamma}-\tilde{T}_{\alpha}^{\gamma} T_{\alpha}^{\delta}=\left(1-e^{2 i h}\right) \sum_{\gamma^{\prime}=\sup (1, \gamma+\delta-N-1)}^{\gamma-1}\left(\widetilde{T}_{\alpha}^{\gamma^{\prime}} T_{\alpha}^{\gamma+\delta-\gamma^{\prime}}-\widetilde{T}_{\alpha}^{\gamma+\delta-\gamma^{\prime}} T_{\alpha}^{\gamma^{\prime}}\right) .
$$

(For $\gamma=1$ we get $\tilde{T}_{\alpha}^{1} T_{\alpha}^{\gamma}=\widetilde{T}_{\alpha}^{\gamma} T_{\alpha}^{1}$.) The general solution of these relations is simply:

$$
\tilde{T}_{\alpha}^{\delta} T_{\alpha}^{\gamma}=\widetilde{T}_{\alpha}^{\gamma} T_{\alpha}^{\delta} .
$$

The situation is unfortunately more complicated for $\alpha \neq \beta$. We must distinguish the three cases $\gamma<\delta, \gamma=\delta$, and $\gamma>\delta$, and treat the cases $\gamma$ or $\delta=1$ separately. Choose $\beta>\alpha$ once for all. After simple algebraic manipulations, we arrive at the following recursive relations. Define

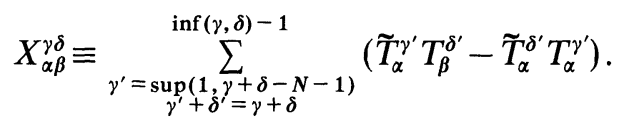

[It follows from Eq. (B.4) that $X_{\alpha \alpha}^{\gamma \delta}=0$.] The recursion is given by:

1. $\gamma=\delta$.

$$
e^{2 i h} \widetilde{T}_{\beta}^{\gamma} T_{\alpha}^{\gamma}-\widetilde{T}_{\beta-1}^{\gamma} T_{\alpha+1}^{\gamma}=\widetilde{T}_{\alpha}^{\gamma} T_{\beta}^{\gamma}-e^{2 i h} \widetilde{T}_{\alpha+1}^{\gamma} T_{\beta-1}^{\gamma}+\left(e^{2 i h}-1\right)\left(X_{\alpha \beta}^{\gamma \gamma}-X_{\alpha+1, \beta-1}^{\gamma \gamma}\right),
$$

with the limiting case $\beta=\alpha+1$,

$$
e^{2 i h} \widetilde{T}_{\alpha+1}^{\gamma} T_{\alpha}^{\gamma}=\tilde{T}_{\alpha}^{\gamma} T_{\alpha+1}^{\gamma}+\left(e^{2 i h}-1\right) X_{\alpha \alpha+1}^{\gamma \gamma} ;
$$

2. $\gamma<\delta$.

$$
\begin{aligned}
e^{2 i n} \widetilde{T}_{\beta}^{\gamma} T_{\alpha}^{\delta}-\widetilde{T}_{\beta-1}^{\gamma} T_{\alpha+1}^{\delta}= & \widetilde{T}_{\alpha}^{\delta} T_{\beta}^{\gamma}-\widetilde{T}_{\alpha+1}^{\delta} T_{\beta-1}^{\gamma}-\left(e^{2 i h}-1\right) \widetilde{T}_{\alpha+1}^{\gamma} T_{\beta-1}^{\delta} \\
& +\left(e^{2 i h}-1\right)\left(X_{\alpha \beta}^{\gamma \delta}-X_{\alpha+1, \beta-1}^{\gamma \delta}\right),
\end{aligned}
$$

with the limiting case $\beta=\alpha+1$,

$$
e^{2 i h} \widetilde{T}_{\alpha+1}^{\gamma} T_{\alpha}^{\delta}=\widetilde{T}_{\alpha}^{\delta} T_{\alpha+1}^{\gamma}+\left(e^{2 i h}-1\right) X_{\alpha \alpha+1}^{\gamma \delta} ;
$$


3. $\gamma>\delta$.

$$
\begin{aligned}
e^{2 i h}\left(\widetilde{T}_{\beta}^{\gamma} T_{\alpha}^{\delta}-\widetilde{T}_{\alpha}^{\delta} T_{\beta}^{\gamma}\right) & -\left(\widetilde{T}_{\beta-1}^{\gamma} T_{\alpha+1}^{\delta}-\widetilde{T}_{\alpha+1}^{\delta} T_{\beta-1}^{\gamma}\right)=-\left(e^{2 i h}-1\right)\left(\widetilde{T}_{\alpha}^{\gamma} T_{\beta}^{\delta}+\widetilde{T}_{\alpha+1}^{\delta} T_{\beta-1}^{\gamma}\right) \\
& +\left(e^{2 i h}-1\right)\left(X_{\alpha \beta}^{\gamma \delta}-X_{\alpha+1, \beta-1}^{\gamma \delta}\right),
\end{aligned}
$$

with the limiting case $\beta=\alpha+1$,

$$
e^{2 i h}\left(\widetilde{T}_{\alpha+1}^{\gamma} T_{\alpha}^{\delta}-\widetilde{T}_{\alpha}^{\delta} T_{\alpha+1}^{\gamma}\right)=-\left(e^{2 i h}-1\right) \widetilde{T}_{\alpha}^{\gamma} T_{\alpha+1}^{\delta}+\left(e^{2 i h}-1\right) X_{\alpha \alpha+1}^{\gamma \delta} .
$$

Starting from the relations (B.1) for $\alpha>\beta$ we get the same results as expected.

These relations may be easily used to express $\widetilde{T}_{\alpha+r}^{\gamma} T_{\alpha}^{\delta}$ in terms of $\widetilde{T}_{\alpha+s}^{\gamma^{\prime}} T_{\alpha+r-s}^{\alpha+\delta-\gamma^{\prime}}$, with $s<\left[\frac{r}{2}\right]$. This establishes the table of multiplication.

For comparison, we note that the same calculation applied to the standard exchange matrix of $S L(N)_{q}$ Eq. (48) gives, for $\beta>\alpha$,

$$
\begin{aligned}
T_{\beta}^{\gamma} T_{\alpha}^{\delta} & =T_{\alpha}^{\delta} T_{\beta}^{\gamma} & & \delta>\gamma, \\
T_{\beta}^{\gamma} T_{\alpha}^{\gamma} & =e^{-i h} T_{\alpha}^{\gamma} T_{\beta}^{\gamma} & & \delta=\gamma, \\
T_{\beta}^{\gamma} T_{\alpha}^{\delta}-T_{\alpha}^{\delta} T_{\beta}^{\gamma} & =\left(e^{-i h}-e^{i h}\right) T_{\alpha}^{\gamma} T_{\beta}^{\delta} & & \delta<\gamma .
\end{aligned}
$$

Moreover, if $\beta=\alpha$ and $\delta>\gamma$, one has:

$$
T_{\alpha}^{\delta} T_{\alpha}^{\gamma}=e^{-i h} T_{\alpha}^{\gamma} T_{\alpha}^{\delta}
$$

\section{References}

1. Babelon, O.: Phys. Lett. B 215, 523 (1988)

2. Gervais, J.-L.: The quantum group structure of $2 \mathrm{D}$ gravity and minimal models. Preprint Commun. Math. Phys. 130, 257 (1990)

3. For recent reviews see J.-L. Gervais: Nucl. Phys. B (Proc. Supp.) 5B, 119 (1988). General approach to two-dimensional critical models. In: Common trends in particle and condensed matter physics, Cargèse 1988, Phys. Rep. to be published

Bilal, A., Gervais, J.-L.: Conference Proceedings, "Infinite dimensional Lie algebras and Lie groups," Marseille 1988, World-Scientific

4. Fatteev, V., Zamolodchikov, A.B.: Nucl. Phys. B 280 [FS18], 644 (1987)

Fatteev, V., Lyakianov, S.: Int. J. Mod. Phys. A 3, 507 (1988)

5. Bilal, A., Gervais, J.-L.: Phys. Lett. B 206, 412 (1988)

Bilal, A., Gervais, J.-L.: Nucl. Phys. B 314, 646 (1989)

Bilal, A., Gervais, J.-L.: Nucl. Phys. B 318, 579 (1989)

6. See, e.g. Takhtadjan, L.A.: Quantum groups and integrable models. Preprint Taginuchi lecture notes, 1988

Communicated by L. Alvarez-Gaumé 